\title{
Evaluating Extracellular Matrix influence on adherent cell signaling by Cold Trypsin Phosphorylation-specific Flow Cytometry
}

Iren Abrahamsen and James B Lorens ${ }^{*}$

\begin{abstract}
Background: Tissue microenvironments comprise different extracellular matrix (ECM) proteins that regulate cellular responsiveness to growth factors. In vitro culture of adherent cells on ECM-coated substrata is commonly used to study microenvironmental influence on specific cell signaling responses. Phosphorylation-specific flow cytometry can be utilized to quantify intracellular phosphorylation-dependent signaling events in single cells. However this approach necessitates trypsinization of adherent cells to accommodate flow cytometric analysis. Trypsin is a potent activator of cell signaling and can obscure signal transduction events induced by other factors.

Results: To address this we developed a cold trypsin-phosphorylation-specific flow cytometry protocol, where adherent cells are prepared for flow cytometric analysis on ice $\left(\sim 0^{\circ} \mathrm{C}\right)$, a temperature where trypsin retains activity but where intracellular kinases are inactive. We show that this straightforward approach can be used to quantify intracellular pERK levels in single adherent primary human vascular smooth muscle cells grown on different ECM.

Conclusions: Exploiting the limited temperature dependence of trypsin facilitated development of a generally applicable phosphorylation-specific flow cytometry method for analysis of adherent cell types including primary patient derived cells. We demonstrate the utility of cold trypsin-phosphorylation-specific flow cytometry analysis of cell signaling to measure microenvironmental influence in single adherent cells.
\end{abstract}

Keywords: Flow cytometry, Phosphorylation, Cell signaling, Extracellular matrix

\section{Background}

The signaling pathways that drive different normal and pathological cellular responses are controlled by microenvironmental cues, including cell-cell interactions, ECMintegrin engagement and activation of receptor tyrosine kinases by growth factors. This intracellular signal transduction creates unique response-dependent epitopes that can be detected by specific antibodies providing the opportunity to measure signaling events by various immuno-techniques. Phosphorylation-specific flow cytometry enables measurement of the phosphorylation status of multiple intracellular signaling proteins at a single-cell level [1-3]. However, the laminar fluidic flow systems of standard flow cytometers require that cells are in suspension and a near-spherical configuration for effective

\footnotetext{
* Correspondence: jim.lorens@biomed.uib.no

Department of Biomedicine, University of Bergen, Jonas Lies Vei 91, 5009 Bergen, Norway
}

analysis. To allow the adaptation of phosphorylationspecific flow cytometry approach to study primary adherent human cells, we took advantage of the broad temperature-independent activity of trypsin to allow proteolytic release of single adherent cells at low temperatures. We show that cold-trypsin phosphorylation-specific flow cytometry analysis can be used to quantify phosphorylated ERK (pERK) levels in single human pulmonary artery-derived smooth muscle cells (PaSMC) exposed to different ECM proteins.

\section{Methods \\ Cold trypsin phosphorylation-specific flow cytometry analysis of adherent cells}

Low passage $(<10)$ PaSMCs (Lonza, Cologne, Germany) were grown at $37^{\circ} \mathrm{C}, 5 \% \mathrm{CO}_{2}$ in human fibroblast growth factor (hFGF)-supplemented smooth muscle growth medium-2 (SmGM-2; Lonza, Cologne, Germany) on ECM protein coated tissue culture plates until about

\section{Biomed Central}


$80 \%$ confluence. Tissue culture plates were coated by passive adsorption with collagen I $(0.1 \mathrm{mg} / \mathrm{ml})$, fibronectin $(15 \mu \mathrm{g} / \mathrm{ml})$ and laminin $(15 \mu \mathrm{g} / \mathrm{ml})$ (Sigma-Aldrich, St. Louis, MO, USA). PaSMC were serum starved overnight prior to stimulation with $50 \mathrm{nM}$ phorbol-12-myristate-13-acetate (PMA; Sigma-Aldrich, St. Louis, MO, USA) for 5 minutes or $20 \%$ fetal bovine serum (FBS; PAA Laboratories, Pasching, Austria) for 15 minutes. The cells were placed on ice immediately after stimulation to quench cell signaling. The cells were then washed twice with ice cold phosphate buffered saline (PBS; Sigma-Aldrich) before adding ice cold 0.5\% TrypsinEthylenediaminetetraacetic acid (EDTA; Sigma-Aldrich). Culture plates were kept on ice and monitored by microscopy until the cells had completely rounded up and detached from the cell culture dish (approximately 25 minutes). Alternatively, cells were detached with $0.25 \%$ Trypsin-EDTA at $37^{\circ} \mathrm{C}$ for 2 minutes. A $16 \%$ paraformaldehyde (PFA) solution (Electron Microscopy Sciences, Hatfield, PA, USA) was added directly to the single cell suspension to obtain a final concentration of $1.6 \%$ PFA. The cells were incubated in the fixative for 20 minutes at room temperature. The cells were thereafter washed with ice cold PBS and pelleted before they were permeabilized in 100\% ice-cold methanol (SigmaAldrich) overnight at $4{ }^{\circ} \mathrm{C}[2,3]$. To stain the cells for flow cytometry, the samples were first centrifuged at $500 \times \mathrm{g}$ for 5 minutes to remove the methanol and then washed with FACS buffer (1\% BSA in PBS). Cells were then stained with rabbit anti-p44/42 MAPK (ERK1/2) antibody (\#4695 Cell Signaling, Danvers, MA, USA) at a dilution of 1:1000 for 30 minutes at room temperature. The cell suspension was then washed with FACS buffer before goat anti-rabbit IgG-Alexa Fluor 647 (A-21244, Invitrogen; Life Technologies, Grand Island, NY, USA) was added at a concentration of $1: 2500(0.8 \mu \mathrm{g} / \mathrm{mL})$ as a secondary antibody. The cells were incubated for 1 hour in darkness at room temperature before being washed once more with FACS buffer. Samples were run on a BD Accuri C6 (BD Biosciences, San Jose, CA, USA) equipped with $488 \mathrm{~nm}$ and $640 \mathrm{~nm}$ lasers at the University of Bergen Flow Cytometry Core Facility. Daily quality control is performed on the instrument using 8- and 6- peak bead sets. The standard bandpass filter used for Alexa Fluor 647: 675/25 Alexa fluor 647 (excitation max: $640 \mathrm{~nm}$, emission max: $668 \mathrm{~nm}$ ). A minimum of 10,000 events was collected for each sample. The gating strategy comprised a standard FSC/SSC live cell gate, followed by a FSC-A/FSC-H single cell gate to minimize inclusion of debris, dead cells and aggregates in the analysis of Alexa Fluor 647 fluorescence. Alternatively fixable live/ dead dyes may be added to prior to PFA treatment to enhance discrimination of dead cells in the analysis. Flow data analysis and median fluorescence intensity (MFI) calculations were performed using FlowJo (TreeStar Inc., Ashland, OR, USA). All fluorescence values were corrected by subtracting the MFI value of the secondary only goat anti-rabbit IgG-Alexa Fluor 647 staining. Cell viability of trypsinization protocols was evaluated by propidium iodide exclusion $(1 \mathrm{ug} / \mathrm{ml}$ for 5 minutes, room temperature) and flow cytometry analysis (BD FACS Aria II, 532 nm laser; 610/20 BP filter). No difference in the percentage of dead cells within the PIpositive gate $(<3 \%)$ was observed following either warm or cold trypsinization of PaSMC.

\section{Western blot analysis}

PaSMC were grown in ECM protein coated $10 \mathrm{~cm}$ cell culture plates until $80 \%$ confluence, serum starved and treated with $50 \mathrm{nM}$ PMA for 5 minutes or 20\% FBS for 15 minutes at $37^{\circ} \mathrm{C}, 5 \% \mathrm{CO}_{2}$. After stimulation the cells were washed twice with ice cold PBS before $300 \mu \mathrm{L}$ icecold Nonidet-P40 (NP40) lysis buffer was added and cells were scraped off the dish using a cell scraper and transferred to an eppendorf tube and kept on ice for 20 minutes. The samples were sonicated 3 times for 10 seconds and then centrifuged at $16,000 \times \mathrm{g}$ for 5 minutes at $4^{\circ} \mathrm{C}$. Supernatants were transferred to a fresh tube and stored at $-20^{\circ} \mathrm{C}$. The protein concentration was then determined by BCA Protein Assay Kit (Thermo Fisher Scientific, Waltham, MA, USA). XT Sample buffer (Bio Rad, Hercules, CA, USA) was added to the samples and heated at $95^{\circ} \mathrm{C}$ for 10 minutes, cooled down and loaded onto NuPAGE 10\% Bis-Tris gels (Invitrogen, Carlsbad, CA, USA), and run for $50 \mathrm{mi}-$ nutes at 200 Volts in NuPAGE MOPS SDS Running Buffer (Invitrogen). Proteins were transferred to a polyvinylidene fluoride (PVDF) transfer membrane (GE Healthcare, Little Chalfont, Buckinghamshire, UK) for 1 hour at 100 Volts. The membrane was blocked in TBS/T (0.1\% Tween 20) with 5\% (weight/volume) dried skimmed milk powder. After blocking, the membrane was washed 4 times for 5 minutes in TBS/T. The membrane was then incubated with anti-pERK primary antibody (p44/42 MAPK antibody 4695; Cell Signaling) at a dilution of 1:2000. The primary antibody was added in $\mathrm{TBS} / \mathrm{T}$ with $5 \%(\mathrm{w} / \mathrm{v}) \mathrm{BSA}$ and incubated over night at $4^{\circ} \mathrm{C}$. The membrane was then washed 4 times for 5 minutes in TBS/T before staining with Goat AntiRabbit IgG (H+L)-HRP Conjugate 170-6515 (Bio-Rad) secondary antibody at a dilution of 1:10,000. The membrane was incubated with secondary antibody in TBS/T with $5 \%(\mathrm{w} / \mathrm{v})$ dried skimmed milk powder for 1 hour at room temperature before being washed again, 4 times for 5 minutes with TBS/T. Loading control was performed using monoclonal mouse Anti-Human Smooth Muscle Actin Clone 1A4 (Dako, Glostrup, Denmark) with Goat Anti-Mouse IgG (H+L)-HRP Conjugate 170-6516 


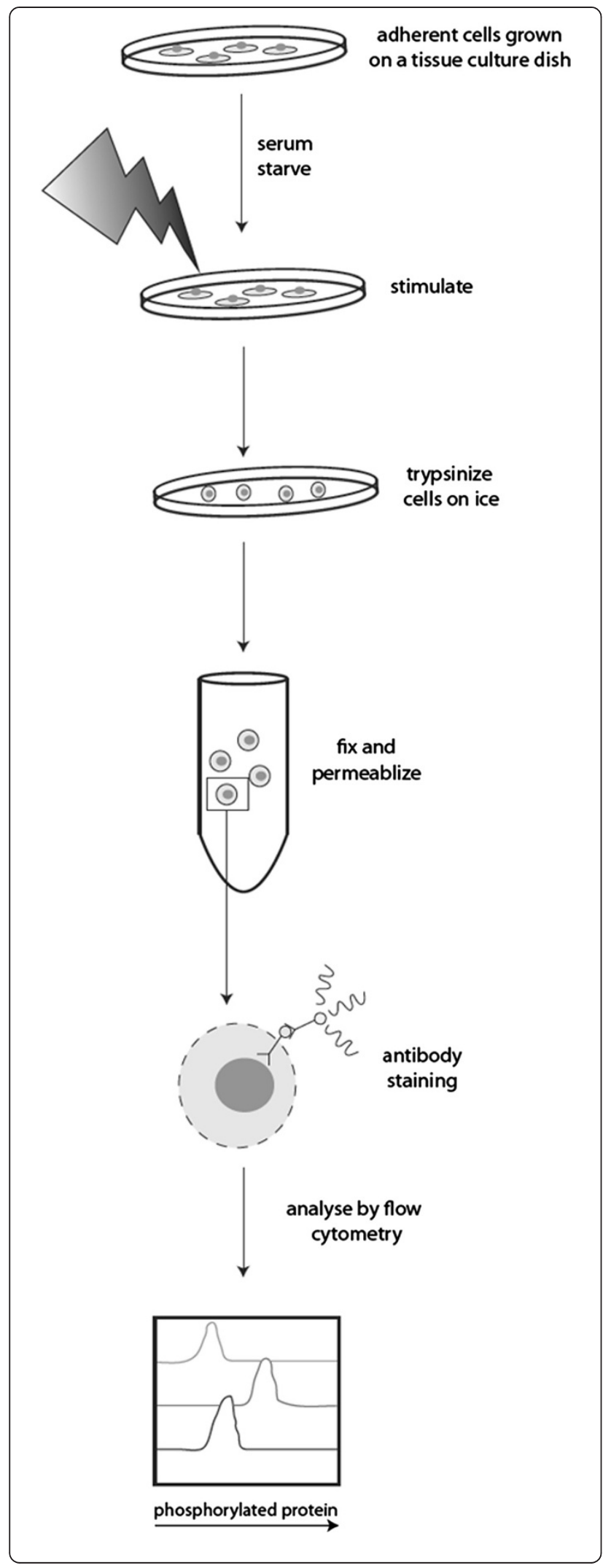

Figure 1 Schematic of the cold trypsin phosphorylation-specific flow cytometry approach. Cells grown on cell culture dishes at $37^{\circ} \mathrm{C}$ are treated with activators for the required time and then immediately placed on ice to quench further cell signaling. Ice cold trypsin is added to the cell culture plates and kept on ice during the trypsinization process in order to minimize intracellular signaling. The cells are then harvested, fixed and permeablized prior to staining with optimized phospho-specific primary antibodies and fluorophore-conjugated secondary reagents. Stained cells are analyzed by flow cytometry to quantify single cell fluorescence values corresponding to phosphorylated levels of intracellular signaling proteins.

(Bio-Rad) as secondary antibody. The membrane was developed in a Chemi Doc XRS (Bio Rad) using Pierce ECL Western Blotting substrate (Thermo Fisher Scientific). Quantification of Western blot bands was conducted using the ImageLab 3.0 software (BioRad). Mean pixel intensity was measured for each band, background was subtracted and samples were normalized to beta-actin.

\section{Results and discussion}

Quantifying pERK in single adherent cells by phosphorylation-specific flow cytometry

Flow cytometry of adherent cells is routinely carried out by first detaching cells from tissue culture dishes or dissociating intact tissues by treating with the serine protease trypsin. Trypsin is known to activate cell signaling via the protease-activator receptor 2 (PAR2), a seven transmembrane-spanning domain $\mathrm{G}$ protein-coupled receptor, by cleaving the amino-terminal exodomain [4]. PAR2 activation stimulates MAP kinase pathway activity in epithelial and smooth muscle cells $[5,6]$. Trypsin activation of PAR2 is also shown to activate MAP phosphatase activity and attenuate pERK level induction by other receptors [6,7]. Hence, trypsin can lead to both potentiated and attenuated pERK levels induced by other receptors.

We reasoned that adherent cells could be prepared for phosphorylation-specific flow cytometry using a cold trypsin solution (Figure 1). Trypsin remains nearly $50 \%$ active at $4{ }^{\circ} \mathrm{C}$, a temperature where intracellular kinases lack activity [8]. Trypsinization of PaSMC on ice $\left(\sim 0^{\circ} \mathrm{C}\right)$ did not adversely affect cell viability (data not shown), consistent with a previous report [9]. Semiconfluent low passage PaSMC cultures were stimulated with PMA at $50 \mathrm{nM}$ for 5 minutes, or with $20 \%$ FBS for 15 minutes and prepared for phosphorylation-specific flow cytometry analysis by trypsinization at $37^{\circ} \mathrm{C}$ or on ice. Cells were kept on ice during the cold trypsin protocol in order to quench cell signaling. PaSMC detached within 25 minutes on ice and within approximately 2 minutes at $37^{\circ} \mathrm{C}$. Detached PaSMC were collected, fixed with paraformaldehyde, permeabilized with methanol and stained with anti-pERK1/2 (phospho- 
Thr202/Tyr204) antibody. Stained cell suspensions were analyzed by flow cytometry to determine pERK levels. In preliminary experiments we noted variation in pERK levels following trypsinization at $37^{\circ} \mathrm{C}$ in unstimulated cell cultures, while basal pERK levels remained low following trypsinization at on ice (data not shown). This is in part due to the rapid detachment of PaSMC at $37^{\circ} \mathrm{C}$. As shown in Figure 2, PaSMC treated with 50 nM PMA and recovered by trypsin treatment at $37^{\circ} \mathrm{C}$ showed a 1.6-fold increase in pERK $(\mathrm{MFI}=14210)$ as compared to the unstimulated cells (MFI=8844). In contrast, pERK levels in cells recovered by cold trypsin showed a 3.6 -fold increase following treatment with PMA (unstimulated, MFI=6806; PMA treated, MFI=24341). Relative pERK activation was 1.5-fold increase by $20 \%$ FBS treatment when trypsinized at $37^{\circ} \mathrm{C}$ (unstimulated, $\mathrm{MFI}=8844$; serum treated, MFI $=13678$ ) and 1.7-fold increase by $20 \%$ FBS treatment with cold trypsin (unstimulated, MFI=6806; serum treated, MFI=11713). In order to verify the specificity of the antiphosphorylated ERK signal detected by phosphorylationspecific flow cytometry, cells were pretreated with an inhibitor of the upstream MAP kinase MEK1 (PD98059) at $30 \mu \mathrm{M}$ for 3 hours. Pretreatment with PD98059 inhibited pERK activation in PaSMCs stimulated with PMA or
A $\quad 0^{\circ} \mathrm{C}$ trypsinization

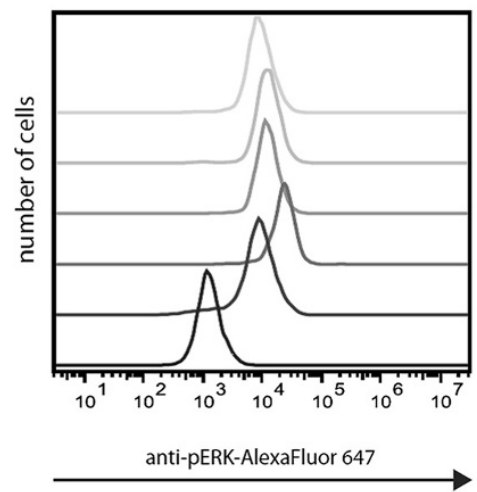

$\mathbf{B}$

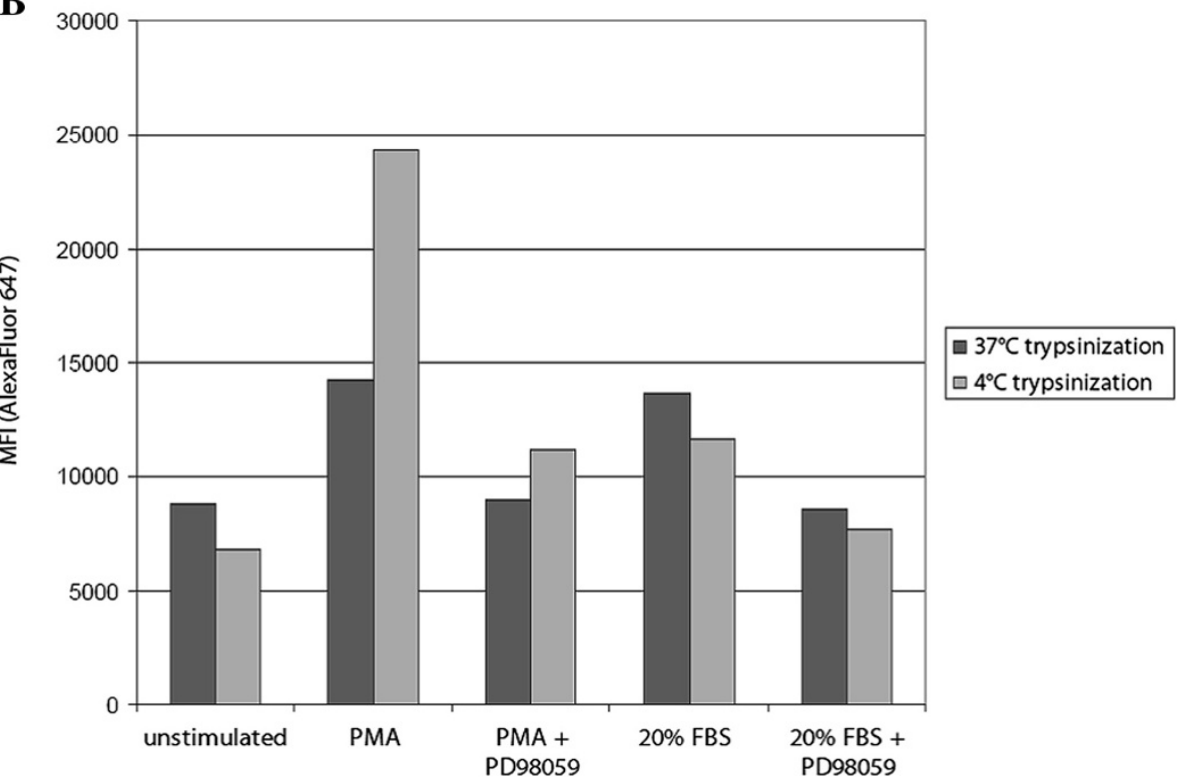

Figure 2 pERK levels are affected by post-treatment trypsinization at different temperatures. PaSMC cultures were stimulated with PMA $50 \mathrm{nM}$ for 5 minutes or $20 \%$ FBS for 15 minutes at $37^{\circ} \mathrm{C}, 5 \% \mathrm{CO}_{2}$ and prepared for phosphorylation-specific flow cytometry analysis by trypsinization at $37^{\circ} \mathrm{C}$ or $0^{\circ} \mathrm{C}$. Cells were pretreated with the MEK1 inhibitor PD98059 at $30 \mu \mathrm{M}$ for 3 hours to inhibit pERK activation. A: Flow cytometry analysis of pERK levels in PMA- and serum-treated PaSMC. Rabbit anti-pERK/goat-anti-rabbit-Alexa Fluor 647 secondary antibody was quantified (10,000 events) and plotted as overlaid histograms of fluorescence versus number of cells. B: Median fluorescence intensity (MFI) measured for PMA- and serum-induced pERK levels following post-treatment trypsinization at $37^{\circ} \mathrm{C}$ or $0^{\circ} \mathrm{C}$. Trypsinization at $37^{\circ} \mathrm{C}$ both increased the basal pERK levels and attenuated the activation of pERK by PMA. Control: Goat-anti-rabbit Alexa Fluor 647 secondary only. (Representative of three independent experiments). 


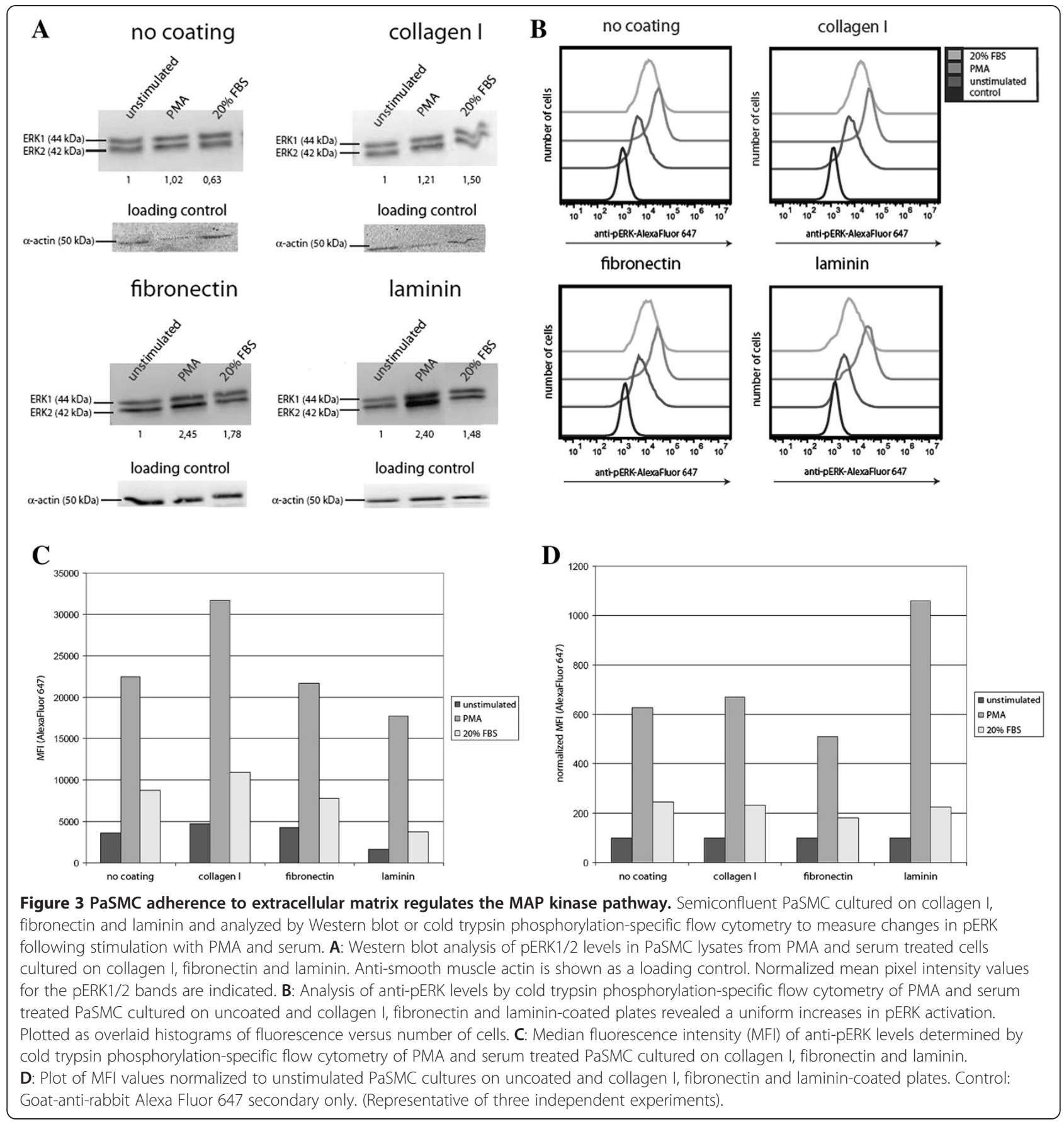

serum stimulation (Figure 2). Collectively, these results indicate that the cold trypsin phosphorylation-specific flow cytometry protocol is a preferable approach to measure cell signaling changes in adherent cells.

\section{Activation of the MAP kinase pathway is regulated by ECM}

Vascular smooth muscle cells (vSMC) are normally surrounded by a laminin-rich basal lamina. During homeostatic or pathological vascular remodeling, vSMCs are exposed to dynamic changes in the local ECM, including loss of basement membrane and synthesis of new ECM components such as fibronectin, that can lead to vSMC proliferation, a major contributor to restenosis, vein graft thickening and atherosclerosis [10-12]. The laminin-rich basal lamina suppresses growth and promotes vSMC differentiation, while fibronectin transitional matrix deposited during vascular remodeling stimulate growth [13].

To address the effect of different ECM proteins on MAP kinase signaling in vSMC, we cultured PaSMCs 
on tissue culture plates coated with collagen I, fibronectin or laminin with growth factor (FGF) and serum supplemented culture medium. Semiconfluent PaSMC cultures were starved overnight and stimulated with PMA or serum. Western blot analysis of PaSMC lysates (Figure 3A) showed relative increases in both pERK1/2 bands at 42 and $44 \mathrm{kDa}$, following treatment with PMA or serum. In order to quantify these ECM-induced changes in MAP kinase pathway activation, we conducted the cold trypsin phosphorylation-specific flow cytometry analysis to measure pERK levels. Histogram plots of the antipERK fluorescence (Figure 3B) revealed a uniform increase in pERK activation in PMA and serum treated PaSMCs cultured on different ECM. Basal pERK levels in PaSMCs cultured on laminin were 2.8 and 2.5 fold less than PaSMC cultured on collagen I or fibronectin respectively (Figure 3C). Treatment with PMA or serum strongly activated pERK under all conditions. Compared to unstimulated cells (MFI= 3576), pERK levels in cells cultured on tissue culture dishes with no coating were upregulated 6.3 and 2.3 fold when treated with PMA (MFI=23700) and serum $(\mathrm{MFI}=8808)$ respectively. For cells growing on collagen I, treatment with PMA and serum upregulated pERK levels 6.7 and 2.3 fold (unstimulated, MFI=4733; PMA treated, MFI=31758; serum treated, MFI=10906). Cells on fibronectin were upregulated 5.1 and 1.8 fold when treated with PMA and serum respectively (unstimulated, MFI $=4249$; PMA treated, MFI=21739; serum treated, MFI=7771), while in cells grown on laminin-coated dishes the upregulation of pERK levels were 10.6 and 2.3 fold when treated with PMA (MFI=17690) or serum (MFI=3775) compared to the unstimulated cells $(\mathrm{MFI}=1668)$. PaSMC cells cultured on collagen and fibronectin display a nearly identical pERK response profile to that of cells cultured on uncoated plates when normalized against unstimulated cells, while cells cultured on laminin displayed an increased relative pERK responsiveness to PMA stimulation (Figure 3D).

\section{Conclusions}

Exploiting the limited temperature dependence of trypsin facilitated development of a generally applicable phosphorylation-specific flow cytometry method facilitating cell signaling analysis in adherent cell types including primary patient derived cells. We demonstrate the utility of cold trypsin-phosphorylation-specific flow cytometry analysis of cell signaling to measure ECM influence in single adherent vSMC. Our results indicate that vSMC adherence to laminin reduces basal pERK levels consistent with suppressed growth, but maintain responsiveness to serum and PKC-dependent signaling.
Flow cytometry analysis offers an attractive alternative to other approaches to multiparametric measurement of phosphorylation-dependent cell signaling events in adherent cells at single cell resolution, such as fluorescence scanning of arrayed cells or high content microscopy-based systems [14]. In particular, phosphorylation-specific flow cytometry offers superior quantification of phospho-epitopes detected by immunofluorescence $[2,3]$. As this sensitivity is achieved at the expense of cell morphological information, an integration of complementary flow cytometry and microscopy approaches is optimal.

\section{Competing interest}

The authors declared that they have no competing interests.

\section{Authors' contributions}

IA performed experiments, data analysis and interpretation and contributed to preparation of the manuscript; JBL conducted data analysis, interpreted data and writing of the manuscript. Both authors read and approved the final manuscript.

\section{Acknowledgements}

Thanks to Sissel Vik Berge, Marianne Enger and Ingrid Strand for excellent technical assistance.

\section{Sources of funding}

Norwegian Research Council grant (182061) to JBL; University of Bergen predoctoral fellowship to IA

Received: 17 April 2013 Accepted: 13 August 2013

Published: 19 August 2013

\section{References}

1. Perez OD, Kinoshita S, Hitoshi Y, Payan DG, Kitamura T, Nolan GP, Lorens JB: Activation of the PKB/AKT pathway by ICAM-2. Immunity 2002, 16:51-65.

2. Perez OD, Nolan GP: Simultaneous measurement of multiple active kinase states using polychromatic flow cytometry. Nat Biotechnol 2002, 20:155-162.

3. Schulz KR, Danna EA, Krutzik PO, Nolan GP: Single-cell phospho-protein analysis by flow cytometry. Curr Protoc Immunol 2011, Chapter 8:11-20. Unit 817.

4. Dery O, Corvera CU, Steinhoff M, Bunnett NW: Proteinase-activated receptors: novel mechanisms of signaling by serine proteases. Am J Physiol 1998, 274:C1429-C1452.

5. Belham CM, Tate RJ, Scott PH, Pemberton AD, Miller HR, Wadsworth RM, Gould GW, Plevin R: Trypsin stimulates proteinase-activated receptor-2dependent and -independent activation of mitogen-activated protein kinases. Biochem J 1996, 320(Pt 3):939-946.

6. Darmoul D, Gratio V, Devaud H, Laburthe M: Protease-activated receptor 2 in colon cancer: trypsin-induced MAPK phosphorylation and cell proliferation are mediated by epidermal growth factor receptor transactivation. J Biol Chem 2004, 279:20927-20934.

7. Namkung W, Yoon JS, Kim KH, Lee MG: PAR2 exerts local protection against acute pancreatitis via modulation of MAP kinase and MAP kinase phosphatase signaling. Am J Physiol Gastrointest Liver Physiol 2008, 295:G886-G894.

8. Matthews PM, Bland JL, Radda GK: The temperature dependence of creatine kinase fluxes in the rat heart. Biochim Biophys Acta 1983, 763:140-146.

9. MCKeehan WL: The effect of temperature during trypsin treatment on viability and multiplication potential of single normal human and chicken fibroblasts. Cell Biol Int Rep 1977, 1:335-343.

10. Haas R, Culp LA: Properties and fate of plasma fibronectin bound to the tissue culture substratum. J Cell Physiol 1982, 113:289-297.

11. Giancotti FG, Ruoslahti E: Integrin signaling. Science 1999, 285:1028-1032. 
12. Clyman RI, McDonald KA, Kramer RH: Integrin receptors on aortic smooth muscle cells mediate adhesion to fibronectin, laminin, and collagen. Circ Res 1990, 67:175-186.

13. Orr AW, Hastings NE, Blackman BR, Wamhoff BR: Complex regulation and function of the inflammatory smooth muscle cell phenotype in atherosclerosis. J Vasc Res 2010, 47:168-180,

14. Zanella F, Lorens JB, Link W: High content screening: seeing is believing. Trends Biotechnol 2010, 28:237-245.

doi:10.1186/1471-2121-14-36

Cite this article as: Abrahamsen and Lorens: Evaluating Extracellular

Matrix influence on adherent cell signaling by Cold Trypsin

Phosphorylation-specific Flow Cytometry. BMC Cell Biology 2013 14:36.

Submit your next manuscript to BioMed Central and take full advantage of:

- Convenient online submission

- Thorough peer review

- No space constraints or color figure charges

- Immediate publication on acceptance

- Inclusion in PubMed, CAS, Scopus and Google Scholar

- Research which is freely available for redistribution

Submit your manuscript at www.biomedcentral.com/submit
(O) Biomed Central 